\title{
COBERTURA TERRITORIAL Y EQUIDAD DISTRIBUCIONAL EN LA RED DE ACCESO PÚBLICO A LA INFORMACIÓN EN LA PROVINCIA DEL CHACO-API-.
}

Ing. Udrízar Lezcano M. Sandra

Grupo de Investigación en Telecomunicaciones Rurales (GTR-UNNE)

Facultad de Ingeniería-UNNE - Av. Las Heras 727, CP.3500 Resistencia, Argentina.

Tel: +54 3722420076 Ext. 143 -Fax: +54 3722428106 sudrizar@ing.unne.edu.ar

Dra. Ramírez Liliana

Instituto de Geografía - Laboratorio de Cartografía Digital

Facultad de Humanidades - UNNE - Av. Las Heras 727, CP.3500 Resistencia, Argentina.

Telefax: +54 3722446958 - Te: +54 03722422257 Iramirez@hum.unne.edu.ar

\section{Resumen}

Una red de Acceso Público a la Información (API) compuesta fundamentalmente por Bibliotecas Populares (BP) y Centros Tecnológicos Comunitarios (CTC) se constituye en instrumento de desarrollo al permitir el acceso colectivo a las TICs, dado que la comunidad puede hacer uso de equipamiento informático y de una conexión a Internet sin tener que asumir los costos involucrados en estos procesos.

En este aporte se analiza la localización de dichos puntos de acceso en la provincia del Chaco, examinándose tanto la cobertura territorial como la equidad distribucional de los mismos empleando SIG y calculando distintos índices de localización y concentración. Los resultados demuestran una desigual distribución de los recursos apreciándose asimismo desorden territorial en la localización de ellos.

Palabras Clave: TIC, Acceso Universal, SIG, Localización.

\section{TERRITORIAL COVERAGE AND DISTRIBUCIONAL EQUITY IN THE PUBLIC ACCESS} NETWORK AT CHACO'S PROVINCE.

\section{Summary}

Public Access Networks to the Information ( PAI ) integrated fundamentally by Public Libraries ( PL ) and Technological Community Centres ( TCC ) constitutes itself in instrument of development when permit the collective access to $\mathrm{t}$ ITC, granted that the community can make use of informationtechnology and connection to Internet without having to assume the costs implicated in these processes.

In this contribution we examine the location of the aforementioned points of access at the Chaco's province, taking an exam so much territorial coverage like distributional equity using SIG and calculating diverse indexes of location and concentration. The results demonstrate an unequal allocation of resources and territorial disorder at their location.

Key Words: ICT, Universal Access, GIS, location.

\section{Sumario}

- Introducción

- Las fuentes de información

- El vínculo entre las Bases de Datos geométrica y de atributos

- La Base Geométrica Nacional y Provincial

- Análisis de la Cobertura Territorial: Localización y Distribución espacial de los CTCs (Centros Tecnológicos Comunitarios) y de las BP (Bibliotecas Populares) en la Provincia del Chaco

- Análisis de la Equidad Distribucional: Índices de desigualdad distribucional y cociente de localización

- Conclusiones

- Bibliografía 


\section{Introducción}

Para que todos los sectores de la sociedad disfruten los beneficios de las Tecnologías de Telecomunicación y la Informática (TIC), es necesario proveer Acceso Universal. Esto no implica necesariamente un teléfono (o en forma más general un punto de acceso) en cada hogar, sino que puede definirse de varias maneras: como un teléfono disponible en un radio de $20 \mathrm{Km}$., o también puede decirse que existe Acceso Universal si para hacer uso del teléfono la persona no deba viajar más de 30 minutos, o incluso puede alcanzarse el objetivo colocando un teléfono en cada localidad de mas de 500 habitantes. (UIT, 1998)

En nuestro país, de manera similar a lo ocurrido en otros países latinoamericanos, se impulsaron planes nacionales como el proyecto de Telecentros Comunitarios (CTC), iniciado por la Secretaría de Comunicaciones de la Nación en el marco del programa "Argentina@Internet.todos" (Dto.1018/98), cuyo objetivo era brindar acceso a la información a los sectores más desfavorecidos mediante la instalación de centros de acceso colectivo. Sin embargo las evidencias indican que las áreas urbanas recibieron la mayor parte de la inversión en infraestructuras, dejando a muchas localidades rurales con pocas o ninguna opción de acceso. (Goussal \& Udrízar Lezcano, 2000)

Además de los 1427 CTC asignados, se encuentran distribuidas en todo el país 1745 Bibliotecas Populares (BP) a las que la Comisión Nacional Protectora de las Bibliotecas Populares (CONABIP) proporcionó equipamiento informático y las que, al igual que los CTC, mediante lo establecido por Res SC 2466/98 se benefician con una bonificación de hasta \$100 en la factura telefónica para conectarse a Internet.

Este trabajo examina la distribución de la red de Acceso Público a la Información (API) compuesta por los CTC y las BP, considerando la dimensión territorial, espacial o geográfica. Llevándose a cabo diferentes tipos de análisis y presentándose imágenes e índices ilustrativos. En este sentido los estudios efectuados sobre la relación de la infraestructura de telecomunicaciones y la economía regional sugieren que la carencia de la misma puede impedir el desarrollo local y regional (Parker, 2000; Lentz \& Oden, 2001).

Se utilizó como base territorial a las 25 unidades departamentales que conforman la Provincia del Chaco, a los 88 puntos que representan las localidades relevadas en el Censo Nacional de Población, Hogares y Viviendas 2001 y los 68 municipios; en conjunción con una base de datos con información relativa a la presencia de puntos de acceso público (BP y CTC) como así también las opciones de conectividad existente en las mismas. Empleándose como herramienta de análisis espacial los Sistemas de Información Geográfica (en adelante SIG).

Las variables que permiten evaluar el acceso pueden resumirse en tres ejes principales:

$\checkmark \quad$ cobertura territorial y/o geográfica;

$\checkmark \quad$ equidad distribucional; $y$

$\checkmark \quad$ asequibilidad económica

La cobertura territorial hace referencia a la existencia de al menos una facilidad en cada localidad o asentamiento poblacional; la equidad distribucional implica la necesidad de que las localidades de menor tamaño o mas alejadas sean prioritarias en cuanto al número de puntos de acceso disponibles y en lo posible no se observen regiones saturadas y otras aisladas; y la asequibilidad económica involucra las políticas gubernamentales destinadas a facilitar el acceso a quienes no pueden afrontar los costos que el mismo conlleva, esto implica la posibilidad de conectarse con tarifas preferenciales.

\section{Las fuentes de información}

Para los análisis efectuados en este trabajo se toma como insumo de partida una base estadística denominada Base de Datos de Acceso Público a la Información (BADAP) que cuenta con información desagregada de sistemas de acceso público y referencias, que cubre un total de 3259 puntos poblados homogéneos de todo el país provista por el Censo Nacional de Población, Hogares y Vivienda 2001. La misma se originó en tareas de evaluación de Telecentros Comunitarios (Proyecto TCR-Fase 3) que el Grupo de Investigación en Telecomunicaciones Rurales (GTR-UNNE) desarrollara mediante estudios de casos. Esta recopilación de datos se utiliza actualmente para 
efectuar diversos estudios, como ser análisis del patrón de localización y evaluación de proyectos de conectividad a Internet o acceso a la información en pequeñas comunidades.

Algunas de las fuentes de datos volcadas en la BADAP son:

$\checkmark$ Censo Nacional de Población, Hogares y Viviendas 2001-desagregado por localidad

$\checkmark$ 2. Listados de asignación de telecentros comunitaros del programa CTCArgentina@internet.todos (Res SC 920/99 y 4167/99-Boletín Oficial de la Nación)

$\checkmark$ 3. Bibliotecas Populares de la Comisión Nacional Protectora de Bibliotecas Populares (CONABIP)

La actualización de esta Base de Datos insumió varios meses de trabajo, efectuándose una fracción importante de la carga y verificación de los datos en forma manual.

En dicha base las localidades censales se encuentran clasificadas por letras desde la $\mathrm{A}$ a la $\mathrm{H}$ de acuerdo con el número de habitantes según se muestra en la siguiente tabla:

\begin{tabular}{|l|c|}
\hline Localidades Urbanas por $N^{\circ}$ de habitantes & Tamaño \\
\hline $1,000,000$ y + & A \\
\hline 500,000 a 999,999 & B \\
\hline 100,000 a 499,999 & $\mathrm{C}$ \\
\hline 50,000 a 99,999 & $\mathrm{D}$ \\
\hline 10,000 a 49,999 & $\mathrm{E}$ \\
\hline 2,000 a 9,999 & $\mathrm{~F}$ \\
\hline 1,000 a 1,999 & $\mathrm{G}$ \\
\hline 500 a 999 & $\mathrm{H}$ \\
\hline Menos de 500 hab. & Rural \\
\hline
\end{tabular}

Tabla 1

\section{El vínculo entre las Bases de Datos geométrica y de atributos}

La resolución de problemas de localización en un territorio se plantea inicialmente, en entornos geométricos. Hacia la década de los '90 la vertiginosa difusión de los Sistemas de Información Geográfica y el uso de los mismos determina una demanda por parte de los usuarios en relación con la necesidad de que aquellos problemas de localización resueltos en entornos geométricos sean incorporados como funciones de los SIG. Esta demanda determinó que rápidamente los SIG se puedan utilizar, con gran provecho, tanto en la resolución de problemas de índole espacial como en la propuesta de proyectos de planificación territorial. Entre las funciones principales se encuentran las de análisis de la accesibilidad que permite realizar estudios de localización y análisis de distribución de servicios en el espacio considerando la población que los necesita. Para ello, estas tecnologías están dotadas de numerosos medios para el análisis de la información geográfica y, sobre todo, para la combinación de diferentes conjuntos de datos, de modo que se obtengan nuevos resultados y mejor información sobre la realidad espacial estudiada (Bosque Sendra et al., 2000). En este sentido en los últimos años los SIG han sido complementados con los SADE, Sistemas de Ayuda a la Decisión Espacial (SSDS, Spatial Decision Support Systems), que permiten obtener decisiones mejor fundadas en diversas cuestiones de naturaleza territorial, entre ellas la localización óptima de equipamientos.

Para abordar cualquier tipo de análisis espacial mediante SIG es preciso disponer de una base de datos que incluya dos componentes: una componente espacial o base de datos gráfica y una componente temática o base de datos de atributos. En nuestro caso la componente espacial debe estar compuesta por entidades poligonales que representan a los departamentos, entidades lineales que representan a la red vial (en particular de la Provincia del Chaco) y entidades puntuales que representan a los asentamientos de población en el territorio. A continuación se describirán las características de las bases de datos nacional y provincial con la que hemos trabajado. 


\section{La Base Geométrica Nacional y Provincial}

Si bien este aporte refiere al análisis de la situación que se presenta en el territorio chaqueño, el proyecto de investigación que la enmarca tiene como espacio de estudio a todo el territorio nacional por ello, en este apartado describiremos las labores realizadas para adecuar e integrar la información de todo el país, ya que una vez logrado ello, fue posible seleccionar la provincia del Chaco y efectuar los análisis correspondientes.

Para iniciar el estudio partimos de una base nacional compuesta por las provincias y departamentos de la República Argentina preparada para el año 1991, en ocasión del Censo Nacional de Población y Vivienda que se llevó a cabo en ese año ${ }^{i}$. Esta base contaba con 524 unidades departamentales y 1805 localidades. Si bien esta información constituyó el punto de partida fue necesario adaptarla, actualizarla y alcanzar una base que, en relación a los intereses propios del trabajo a desarrollar, nos resultara provechosa.

El paso inicial, luego de examinar la información geográfica disponible, fue el de actualizar la misma, así las unidades departamentales que en el presente tiene nuestro país alcanzan a 532 , hecho que nos llevó a verificar la base y observar no sólo que se añadieron distritos departamentales (como en el caso de la Provincia del Chaco), sino que además algunos se separaron y otros se fusionaron, en especial en la Provincia de Buenos Aires. No obstante analizar el problema de la localización de servicios de acceso a la información pública requiere de entidades puntuales, en este caso se partió de una base que contenía 1805 localidades $^{\text {ii }}$, que, al igual que el caso anterior se refería a localidades censadas en oportunidad del Censo Nacional de Población y Viviendas de 1991. Actualizar esta base implicó añadir a las existentes 1320 localidades distribuidas en el territorio, de manera que contamos con una base gráfica constituida por 3125 puntos que representan asentamientos de población. Esta labor, sin duda, ha sido la más ardua, laboriosa y la que demandó mayor tiempo ya que fue necesario encontrar la localización de los más de mil trescientos asentamientos en diversas fuentes de información, en especial las cartas topográficas del Instituto Geográfico Militar y en diversos sitios Web que cuentan con información confiable acerca de la localización precisa de los puntos de acceso. ${ }^{i i i}$

Finalmente se logró alcanzar lo que se muestra en la figura siguiente. Allí se puede observar la base gráfica nacional, compuesta por 532 departamentos y 3125 asentamientos, y la base gráfica provincial integrada por 25 departamentos y 88 asentamientos.

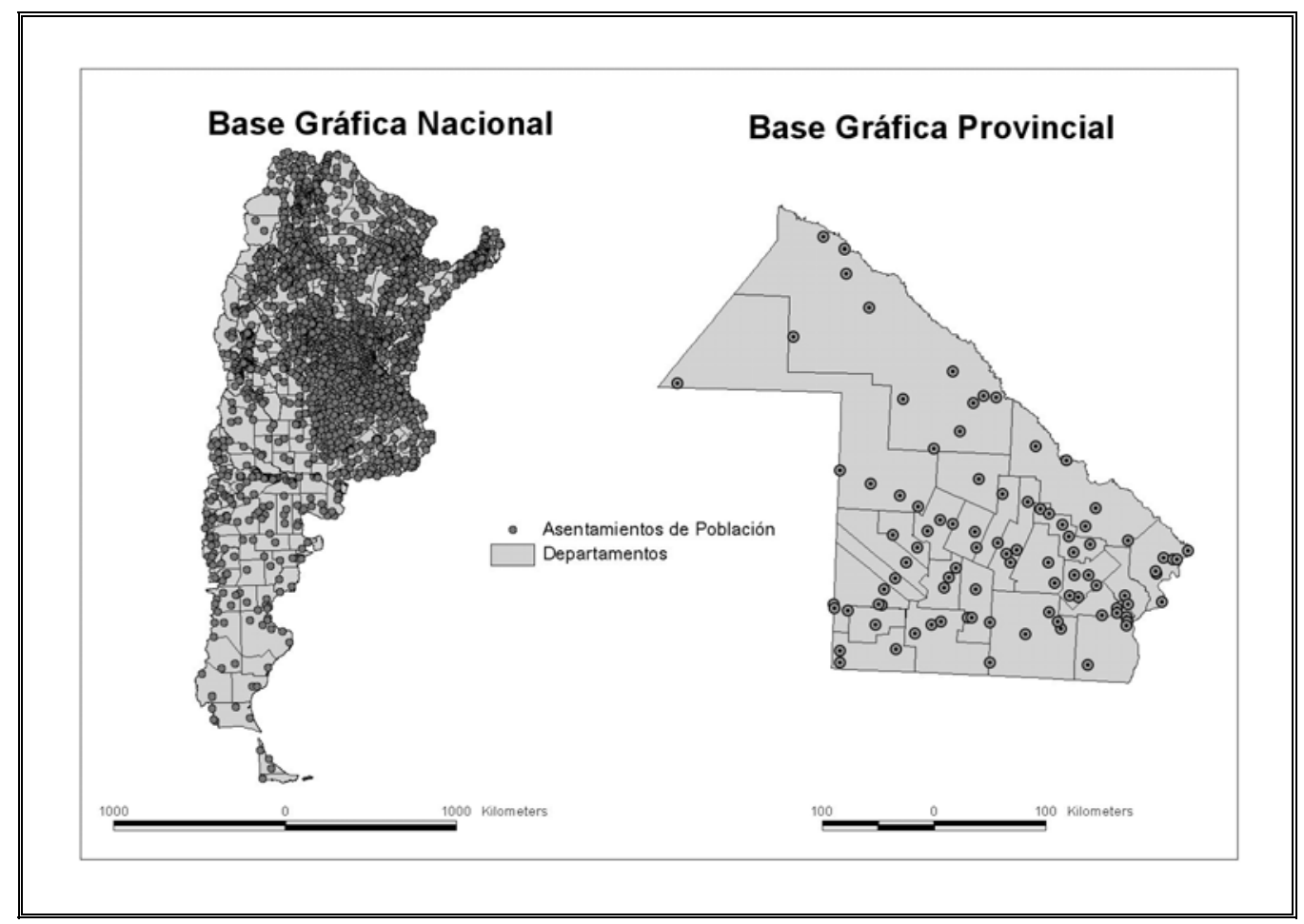

Figura 1 
Análisis de la Cobertura Territorial: Localización y Distribución espacial de los CTCs (Centros Tecnológicos Comunitarios) y de las BP (Bibliotecas Populares) en la Provincia del Chaco

El acceso público a la información es posible si se cuenta con una infraestructura de equipamientos preparados para disponer de recursos que permitan a la población alcanzar determinados servicios. Así para poder analizar el acceso a la información es preciso partir de la localización de instalaciones tales como los Centros Tecnológicos Comunitarios y la Bibliotecas Populares.

De acuerdo con el Censo Nacional de Población, Hogares y Vivienda en el Chaco se han definido 88 asentamientos de población según la cantidad de habitantes, en la tabla 2 se puede apreciar, que no se registran asentamientos de tipo A y B. El examen de las estadísticas, acompañadas de la visualización del gráfico 1, nos permiten señalar que los asentamientos categoría $\mathrm{F}$ son los que predominan en el Chaco, mientras que los asentamientos rurales superan el $20 \%$, ubicándose en segunda posición en lo que a valores relativos se refiere.

\begin{tabular}{|c|c|c|}
\hline \multicolumn{3}{|c|}{ CHACO } \\
\hline Tamaño & Cantidad & Población \\
\hline C & 1 & 359.590 \\
\hline D & 1 & 76.794 \\
\hline E & 10 & 208.616 \\
\hline F & 28 & 139.695 \\
\hline G & 14 & 21.170 \\
\hline H & 14 & 9.507 \\
\hline RURAL & 20 & 4.883 \\
\hline Total & 88 & 820.255 \\
\hline
\end{tabular}

Tabla 2

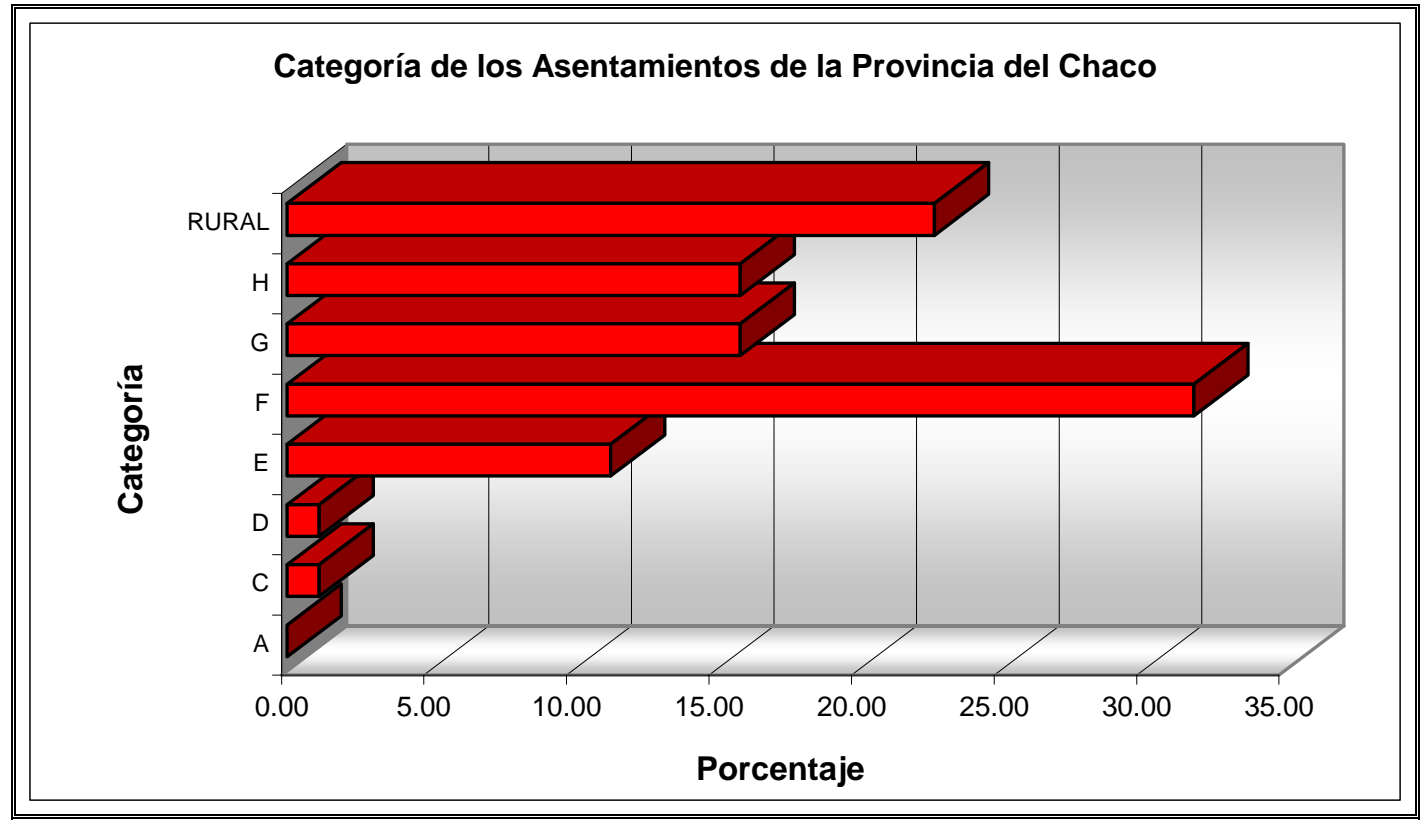

Gráfico 1

Si analizamos el gráfico 2 podemos advertir que su forma es totalmente inversa a la del gráfico 1, con esto queremos decir que si bien el número de asentamientos rurales o de menor rango es mayor, la mayor cantidad de población reside en los pocos asentamientos de categorías más alta. En la provincia analizada el $43,8 \%$ de la población reside en la capital. Esta situación no es nueva 
sino que se va profundizando debido a que las opciones que brinda el medio rural son cada vez menores y ello conduce a que la expulsión de población de este medio hacia la ciudad siga persistiendo.

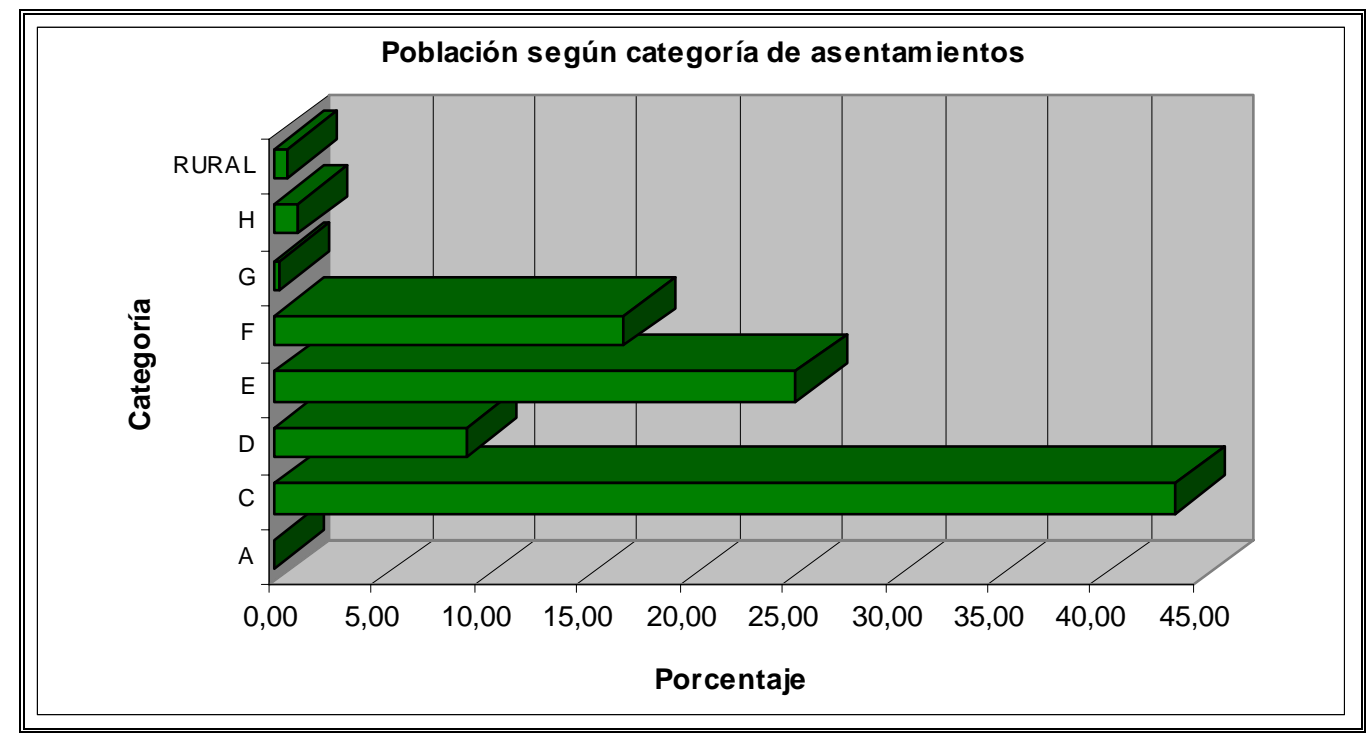

Gráfico 2

Esta presentación del territorio analizado va unida ahora de la dotación de equipamientos o instalaciones como los CTC y las BP. Si bien más adelante aplicaremos determinados índices que nos permitirán valorar esta distribución, como todo análisis de localización hemos iniciado el mismo con los mapas que nos permiten visualizar la misma. En la figura 2 se han incluido la representación de la provincia el Chaco en las cual se muestran todos los asentamientos relevados, localidades con CTC y con BP sobre la base de la división política departamental..

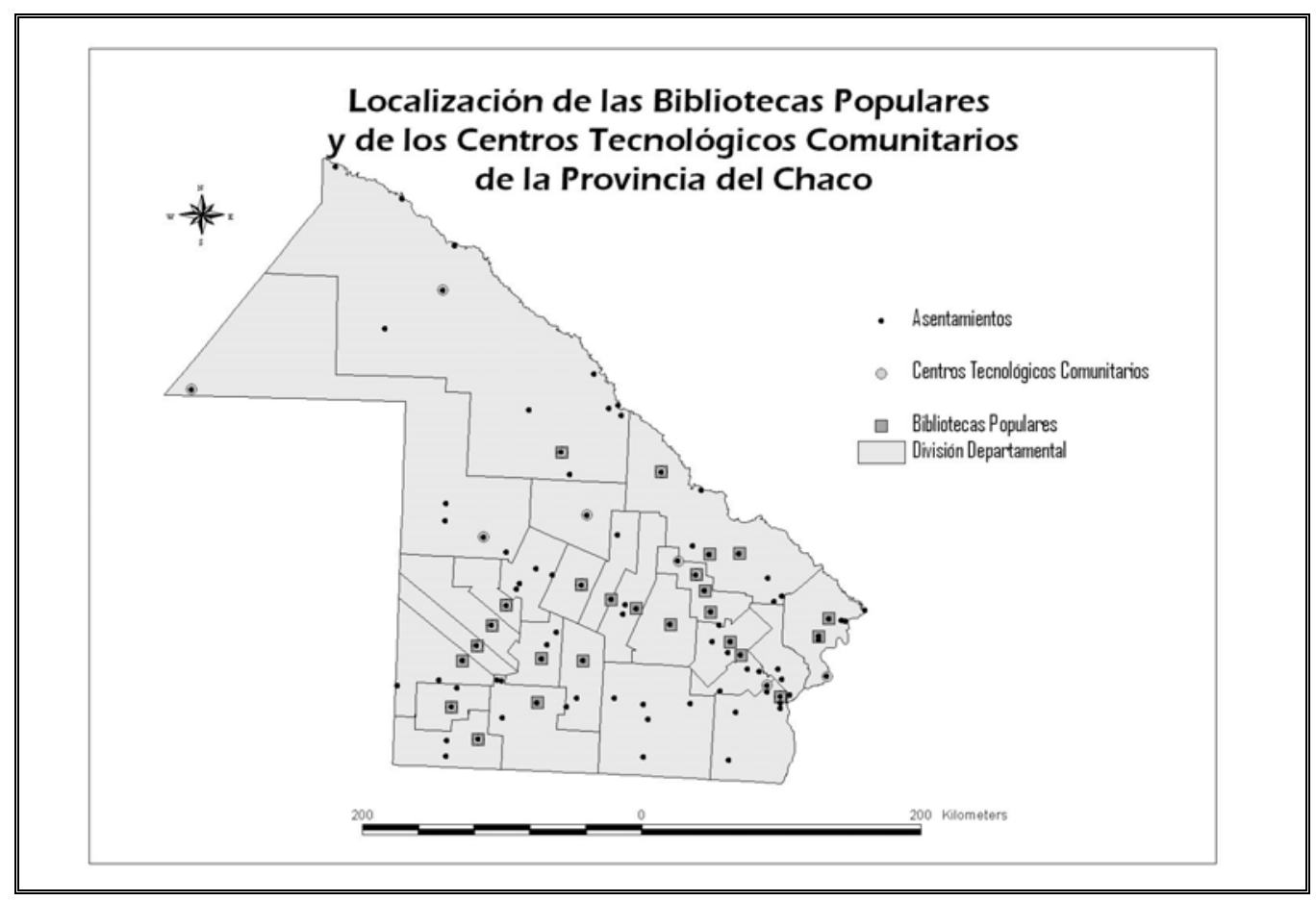

Figura 2 
Podemos apreciar que la representación es bastante elocuente ya que se aprecian los significativos vacíos en cuanto a la localización de equipamientos, hecho que permite adelantar que es elevado el conjunto de población que no alcanza a ser servida por las instalaciones en las que podría tener acceso a la información pública. Así, los sectores más desprovistos corresponden al noroeste y al sureste del territorio

Como podemos ver en la tabla 3 la Provincia del Chaco tiene sólo diecisiete CTC y 25 BP distribuidos entre 88 localidades, lo que representa sólo el 19.32\% del territorio cubierto por algún CTC y $28.41 \%$ con la presencia de al menos una BP. Como se aprecia la cobertura de bibliotecas es siempre mayor que la de los CTC y en ambos casos decrece en áreas de menor tamaño (F-G-H). Existe asimismo un solapamiento de recursos en 6 localidades, resultando igual a 33 el total de localidades con algún punto de acceso (considerando ambas redes en conjunto), lo que significa una cobertura provincial de 37.5\%, valor este muy bajo para el objetivo de Acceso Universal.

\begin{tabular}{|c|c|c|c|c|}
\hline Categoría & $\begin{array}{c}\text { CHACO } \\
\text { Localidades } \\
\text { con CTC }\end{array}$ & $\begin{array}{c}\text { \% de loc. } \\
\text { cubiertas }\end{array}$ & $\begin{array}{c}\mathbf{N}^{\mathbf{0}} \text { de } \\
\text { Localidades } \\
\text { con BP }\end{array}$ & $\begin{array}{c}\text { \% de loc. } \\
\text { cubiertas }\end{array}$ \\
\hline C & 1 & 100 & 1 & 100 \\
\hline D & 1 & 100 & 1 & 100 \\
\hline E & 4 & 40 & 9 & 90 \\
\hline F & 9 & 32.14 & 11 & 39.29 \\
\hline G & 2 & 14.28 & 2 & 14.28 \\
\hline H & -- & --- & 1 & -14 \\
\hline RURAL & -- & ---- & ---- & 28.41 \\
\hline Total & 17 & 19.32 & 25 & \\
\hline
\end{tabular}

Tabla 3

\section{Análisis de la Equidad Distribucional: Índices de desigualdad distribucional y cociente de localización}

No sólo es importante saber cuántas localidades poseen al menos un CTC y/o una BP, sino que es preciso saber cómo se distribuyen lo que daría cuenta de la equidad distribucional. Este principio de localización es muy relevante ya que cuando nos referimos a servicios públicos, ya lo hemos apuntado, sabemos que su reparto en el espacio debe responder al principio de equidad de modo que la totalidad de la población que necesita y quiere hacer uso de ellos pueda acceder a los mismos de igual manera en igualdad de condiciones.

Existen diferentes índices presentados por distintos autores que nos permiten medir los desequilibrios espaciales y también los que se refieren a la desigual concentración de servicios en los territorios. Para analizar estas cuestiones hemos seleccionado algunos de ellos:

1.- El Coeficiente de Gini es una medida de la desigualdad ideada por el estadístico italiano Corrado Gini. Normalmente se utiliza para medir la desigualdad en los ingresos, pero puede utilizarse para medir cualquier forma de distribución desigual. El coeficiente de Gini es un número entre 0 y 1 , en donde 0 se corresponde con la perfecta igualdad (todos tienen los mismos ingresos/recursos/servicios) y 1 se corresponde con la perfecta desigualdad (una persona tiene todos los ingresos/recursos/servicios y todos los demás ninguno). Matemáticamente el coeficiente de Gini se calcula como el promedio aritmético de los valores absolutos de las diferencias entre los pares de valores a comparar, dividido por el valor de referencia o promedio. Se expresa del siguiente modo:

$$
G=2 \int 0[\alpha-g(\alpha)] d \alpha
$$

2.- El Cociente de localización, señalado por JOSEPH y PHILLIPS en 1984, es una magnitud que valora las diferencias inter-regionales en un mismo momento y también las diversidades en el tiempo de una misma región; los resultados obtenidos en este caso fluctúan entre 0 y el infinito, el 
valor 1 es el que indica el equilibrio entre los recursos ofrecidos y los usuarios demandantes ${ }^{\text {iv }}$. En este caso la expresión es la que sigue:

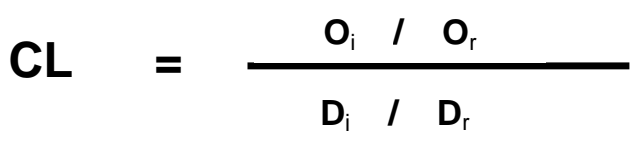

siendo:

$\mathrm{CL}=$ Cociente de Localización referido a un determinado punto o área.

$\mathrm{O}_{i}=$ Oferta existente en un punto de oferta.

$\mathrm{O}_{\mathrm{r}}=$ Oferta en todos los puntos de oferta existentes en la red.

$D_{i}=$ Demanda existente en un punto de demanda.

$D_{r}=$ Demanda en todos los puntos de demanda existentes en la red.

Si aplicamos cada uno de éstos índices a nuestros intereses particulares entonces podemos abonar y profundizar en el tema de la equidad territorial o justicia espacial principio rector de la distribución de equipamientos públicos en el territorio.

La aplicación del Índice de Gini se efectuó en relación a las unidades departamentales por un lado y a los asentamientos de población, por otro lado y arrojó los valores indicados en la tablas 4 y 5 , respectivamente.

\begin{tabular}{|c|c|c|c|c|c|c|c|}
\hline & $\begin{array}{c}\text { Total } \\
\text { Dptos }\end{array}$ & $\begin{array}{c}\text { Dptos. } \\
\text { con CTC }\end{array}$ & $\begin{array}{c}\text { Dptos. } \\
\text { con BP }\end{array}$ & $\begin{array}{c}\text { Deptos con } \\
\text { CTC y/o BP }\end{array}$ & $\begin{array}{c}\text { Índice } \\
\text { Gini CTC }\end{array}$ & $\begin{array}{c}\text { Índice } \\
\text { Gini } \\
\text { BP }\end{array}$ & $\begin{array}{c}\text { Índice } \\
\text { de Gini } \\
\text { CTC+BP }\end{array}$ \\
\hline Chaco & 25 & 14 & 18 & 22 & 0.44 & 0.28 & 0.12 \\
\hline
\end{tabular}

Tabla 4

Iniciando el análisis a nivel departamental vemos que el valor obtenido para el Índice de Gini estudiando aisladamente las redes de CTC y las de BP, presenta una mejor situación en cuanto a la dotación de Bibliotecas Populares, ya que registra 0,28, en tanto que en el caso de los CTC el valor es superior alejándose del 0 que representa la situación ideal (tabla 4). Por otro lado si analizamos el valor que registra el Índice para la red completa, es decir adicionando los CTC y las BP, vemos que aparecen 22 jurisdicciones con algún tipo de dotación y ello implica un registro igual a 0,12, más cercano de la situación más satisfactoria (tabla 4).

\begin{tabular}{|l|c|c|c|c|c|c|c|}
\hline & $\begin{array}{l}\text { Total } \\
\text { Asenta } \\
\text { mientos }\end{array}$ & $\begin{array}{c}\text { Asenta } \\
\text { mientos } \\
\text { con CTC }\end{array}$ & $\begin{array}{c}\text { Asenta } \\
\text { mientos } \\
\text { con BP }\end{array}$ & $\begin{array}{c}\text { Asenta } \\
\text { mientos } \\
\text { con CTC } \\
\text { y/o BP }\end{array}$ & $\begin{array}{c}\text { Índice } \\
\text { Gini } \\
\text { CTC }\end{array}$ & $\begin{array}{c}\text { Índice } \\
\text { Gini } \\
B P\end{array}$ & $\begin{array}{c}\text { Índice } \\
\text { Gini } \\
\text { CTC+BP }\end{array}$ \\
\hline Chaco & 88 & 17 & 25 & 32 & 0.81 & 0.72 & 0.64 \\
\hline
\end{tabular}

Tabla 5

La situación es menos alentadora si obtenemos el índice en relación con las ciudades o asentamientos de población (tabla 5), en este caso, los índices arrojan valores de 0,81 y 0,72 si examinamos individualmente a los CTC y a las BP, respectivamente, lo que indica que más de las 3/4 partes de los asentamientos no cuentan con equipamientos que permitan el acceso público a la información. Si consideramos el total de asentamientos que poseen CTC o BP, aquí el Índice registra 0,64 , un valor menor que los anteriores que implica una mejora en general si tenemos en cuenta toda la red de acceso público.

La aplicación del Cociente de Localización por su parte arroja un resultado por cada jurisdicción analizada, en este caso los departamentos, y permite apreciar la dotación de servicios, CTC y BP, que permitirían el acceso público a la información. Esta medida se puede obtener en forma independiente de la demanda que los requiere o en relación a ella como indicamos más arriba, en este apartado mostraremos la aplicación del índice en forma aislada para apreciar áreas con 
exceso de equipamientos y áreas con déficit de ellos, más adelante lo haremos en relación con los usuarios que potencialmente los emplearían.

Recordemos que como se trata de índices dicotómicos, muestran excedentes e insuficiencias de servicios en relación al valor medio provincial, que simboliza el equilibrio y que está señalado por el valor 1. En cuento a la situación de los Centros Tecnológicos Comunitarios se puede apreciar que los dos departamentos del oeste de la Provincia -General Güemes y Almirante Brown- son los más favorecidos, esta situación tiene que ver con que se trata de las dos jurisdicciones más extensas en superficie y que albergan más de un CTC y si tenemos en cuenta que aquí solo se está analizando la presencia o ausencia de servicios, sin considerar ni la población que los emplea, ni la distancia recorrida por las personas o el tiempo empleado para hacerlo, aspectos que abordaremos en otros trabajos, entonces esta situación se presenta como razonable y es por ello que adquieren valores que superan el 1. En contraposición todos aquellos departamentos que no poseen ningún CTC y se localizan al sur tienen valores por debajo del 1.

En el caso de las Bibliotecas Populares, la situación del Chaco es relativamente mejor aunque no por ello plenamente satisfactoria. Existen quince jurisdicciones que revelan un valor idéntico a la media provincial motivo por el cual registran un cociente de localización igual a 1 . Seis departamentos tienen insuficiencia de servicios, mientras que los cuatro restantes manifiestan excesos de los mismos. Insistimos en que esta situación de excesos no significa que se encuentran en una situación satisfactoria, sino que el índice muestra las disparidades intra-regionales que no se condicen con las necesidades de la región o provincia.

En síntesis si no consideramos la población que demanda los servicios vemos que existen situaciones de desequilibrios regionales de asignación de recursos.

Si tenemos en cuenta el estado general de la red de acceso público, es decir la cantidad total de departamentos que tienen CTC y/o BP podemos obtener un Cociente de Localización para cada jurisdicción, así al representarlos cartográficamente podemos visualizar las áreas que tienen excesos y las que registran déficit en cuanto a la dotación de equipamientos que le permitirían el acceso público (figura 3). Recordemos que los valores inferiores a 1 indican el déficit y se muestran en tonalidades más oscuras, mientras que a la inversa las tonalidades más claras señalan las jurisdicciones con excesos. Entre las primeras se advierten tres departamentos con situación más desfavorable Independencia -al oeste-, Tapenagá -al sur- y Primero de Mayo -en el oriente-.

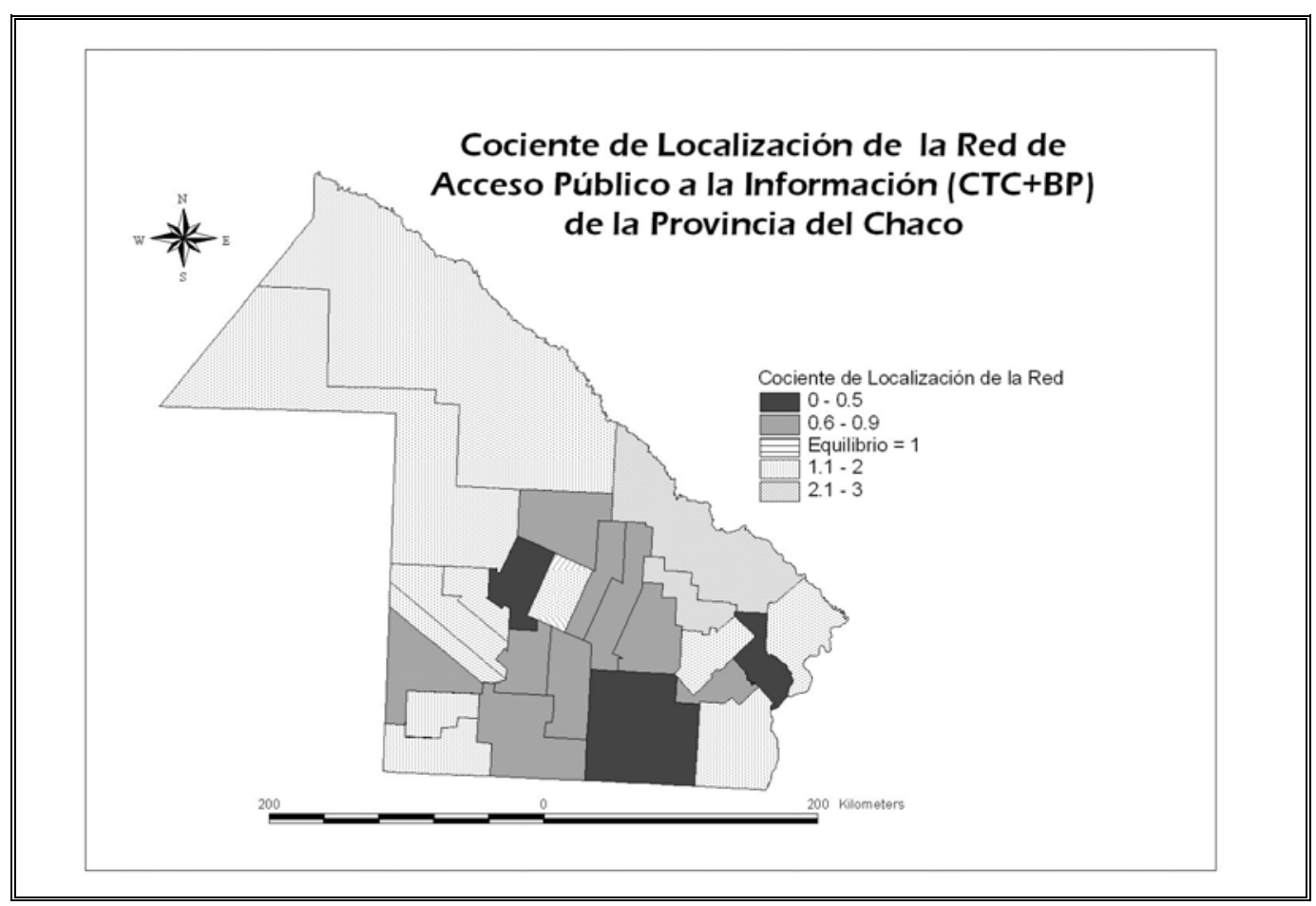

Figura 3 
También con déficit pero menos importantes encontramos a Maipú, Quitilipi, Veinticinco de Mayo, Presidencia de la Plaza, Libertad, Dos de Abril, O’Higgins, Mayor Luis Fontana y San Lorenzo. En oposición Libertador General San Martín y Sargento Cabral en el centro norte de la provincia son los más privilegiados en cuanto a la dotación de equipamientos que permiten el acceso público a la información por parte de los usuarios.

Indaguemos ahora la situación en relación con la población que potencialmente hace uso de estos equipamientos, instalaciones o servicios para poder tener acceso a la red API.

Así la otra forma de aplicar el Cociente de Localización es considerando la proporción de oferta en cada lugar pero en relación con la proporción de demanda insatisfecha en ese mismo punto. De esta forma se analiza la distribución de los servicios con respecto al colectivo de población que potencialmente hace uso de ellos. Es una forma de saber si los equipamientos existentes se distribuyen de manera análoga a la distribución de población en el territorio estudiado. Pero en nuestro análisis a fin de evaluar la equidad distribucional afectaremos el número de habitantes por un coeficiente que nos permita priorizar aquellas localidades de menos de 10,000 habitantes (tamaños F, $\mathrm{G}, \mathrm{H}$ y Rurales). Este procedimiento se emplea dado que cuando se estudia la equidad en una red de Comunicaciones desde el punto de vista geográfico el factor distancia y el factor tiempo adquieren una importancia extrema, pues no se apunta a brindar servicio a la mayor cantidad de población (cosa que se consigue atendiendo varias ciudades grandes ), sino que debe apuntarse a brindar servicio a los clústeres de población que residen más alejados de los grandes centros y que cuentan con menos medios alternativos. Ello en concordancia con lo expuesto en la Declaración de Florianópolis (2000), donde se afirma que aunque el crecimiento económico garantice el progreso de un segmento de la población que ya participa en la sociedad de la información, la no implementación de acciones redistributivas por parte de los estados podría implicar la consolidación de esta nueva forma de exclusión. Así, y a fin de priorizar las poblaciones pequeñas y marginales, hemos ponderado la población de los departamentos de acuerdo con sus Necesidades Básicas Insatisfechas -NBI-, otorgándole un peso o ponderación igual a diez (10) a la jurisdicción con mayor NBI y un valor de uno (1) a la jurisdicción con menor NBI.

Del análisis de la dotación de CTC en relación con la población que potencialmente hace uso de ellos, podemos apreciar que la Provincia del Chaco presenta un déficit bastante marcado en 12 jurisdicciones (48\%) que corresponden al centro y sur del territorio, precisamente allí donde la población es mayoritaria, a ellas se adicionan General San Martín y General Güemes en el norte y noroeste, recordemos que la población ha sido ponderada a través de un factor que potencia las áreas con NBI más elevadas. En el otro extremo de las situaciones podemos decir que los Departamentos Libertad y Dos de Abril son los que se encontrarían, comparativamente hablando, en la mejor posición ya que poseen alrededor de 7 y 8 veces más CTC que el valor medio de la Provincia -siempre en relación con la población atendida-.

En el análisis de la dotación de Bibliotecas Populares considerando la población o demanda que hace uso de los servicios y ponderada además por las Necesidades Básicas Insatisfechas de dicha población, Chaco, tiene 13 departamentos con dotación superior a la media provincial y allí se registran hasta 5 veces más la dotación promedio, correspondiéndole a Sargento Cabral, General Dónovan y Dos de Abril las mejores situaciones; mientras que como vemos en la figura, el noroeste General Güemes, Almirante Brown, Maipú e Independencia- y el sureste -San Fernando, Libertad, Tapenagá y Primero de Mayo-, son las áreas con mayores necesidades de equipamientos. A estas áreas se adicionan Quitilipi, Veinticinco de Mayo y Comandante Fernández en el centro y Mayor Luis Fontana en el sur.

Análogamente al análisis precedente, donde no se incluyó a la población usuaria, aquí hemos obtenido el Cociente de Localización para toda la Red de API y considerando la potencial demanda que emplea estos servicios, siempre ponderada por un indicador que esta en directa relación con las Necesidades Básicas Insatisfechas. En la figura 4 se muestran los resultados del Cociente para cada departamento y ello deja al descubierto las áreas que requerirían un aumento en la dotación de servicios: Independencia, Veinticinco de Mayo, Mayor Luis Fontana, Tapenagá, San Fernando y Primero de Mayo. En cambio los departamentos que cuentan con 3 veces más dotación que el resto de las jurisdicciones, considerando la demanda que emplea los equipamientos, son Dos de Abril, Fray Justo Santa María de Oro, Sargento Cabral y Libertad. 


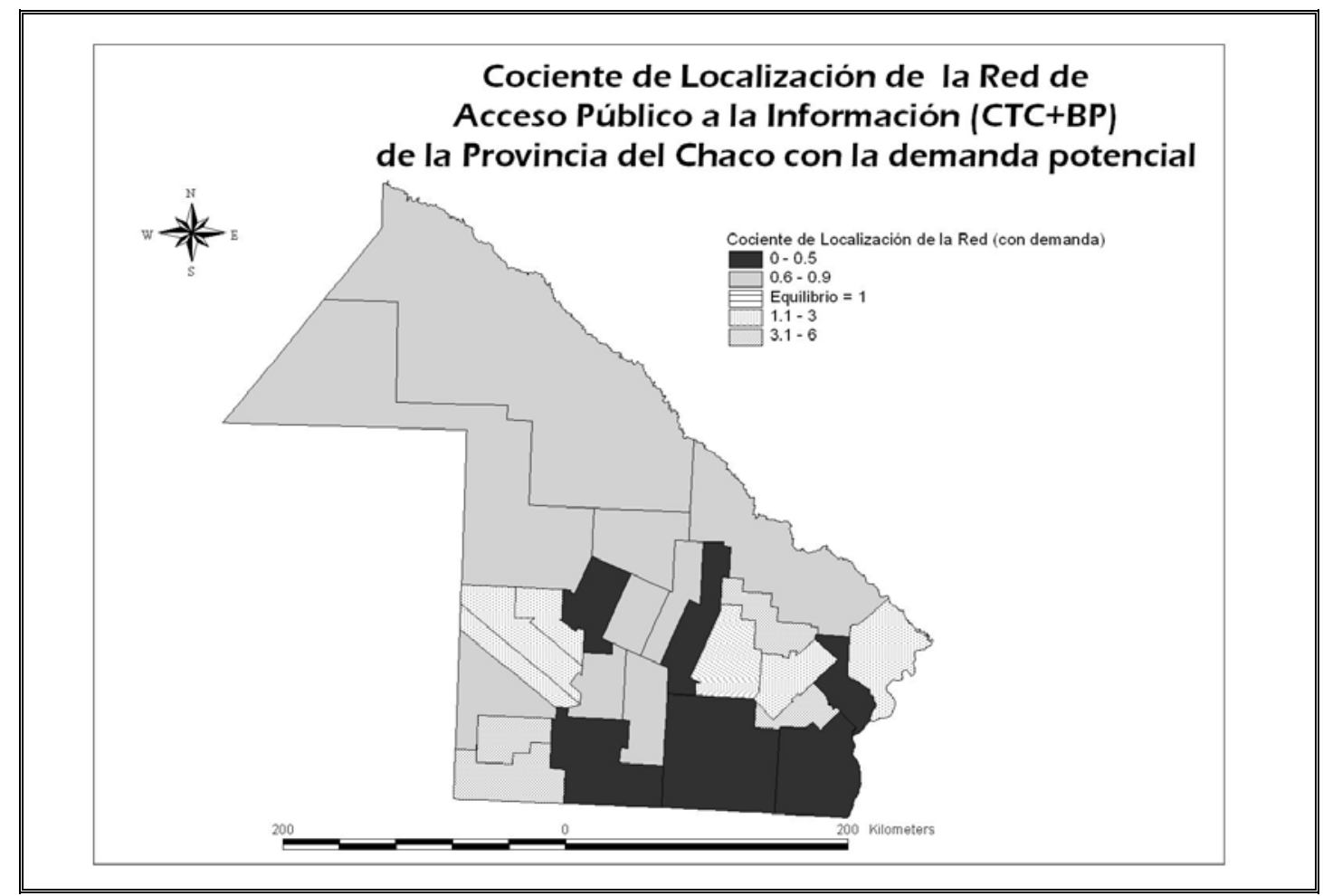

Figura 4

\section{Conclusiones}

Es internacionalmente aceptado que el uso y el conocimiento de las TIC se promueven a través del mero hecho de poseer infraestructuras y brindar capacitación. (Falch, 2003), por ello la importancia de localizarlos adecuadamente fue la arista abordada en este trabajo.

La verdadera dificultad en cualquier red y donde se revela la efectividad y el aporte neto al objetivo de Acceso Universal es el cubrimiento de localidades de menos de 1.000 habitantes (tamaño H), que en nuestra provincia suman el $38.64 \%$, y de los cuales sólo posee cobertura geográfica el $7.14 \%$. Índices tan bajos no hacen más que fortalecer el hecho de que pese a la expansión que significara para el acceso público a la información la red de telecentros comunitarios del plan CTC y la informatización de las BP, las desigualdades de distribución del acceso han permanecido y aún en algún sentido, se han ampliado. (Goussal \& Udrízar Lezcano, 2001)

Dado que las políticas públicas deben orientarse a lograr el bienestar socioeconómico de la población y considerando que las tecnologías de la Información, y la habilidad de usarlas y adaptarlas son los factores críticos en la generación y el acceso a la riqueza, el poder y el conocimiento (Castells, 1998), se torna obvio en base a lo señalado en el presente aporte que las infraestructuras instaladas no respetaron el principio de equidad distributiva y por tanto no resultaron efectivas a la hora de proveer Acceso Universal.

\section{Bibliografía}

- BOSQUE SENDRA, J. et al. (2000), "Hacia un sistema de ayuda a la decisión espacial para la localización de equipamientos". Revista de Estudios Geográficos № 241. 567-598.

- BOSQUE SENDRA, J., (1992). Sistemas de Información Geográfica. Madrid, España Ediciones Rialp S.A., 1992.

- CASTELLS, M. (1998) The end of the millennium. Massachusets: Blackwell.

- Declaración de Florianópolis (2000). Reunión Regional de Tecnología de Información para el Desarrollo. Florianópolis, Santa Catarina, Brasil, 20-21 junio 2000, convocada por la CEPAL (Comisión Económica para América Latina y el Caribe de las Naciones Unidas) http://WWW.ECLAC.ORG/espanol/topicos/lcl1383/indice.htm 
- FALCH, M.; ANYIMADU, A.(2003) "Tele-centres as way of achieving universal access - the case of Ghana". Telecomunications Policy 27. Pp 21-39.

- GOUSSAL, D.;UDRÍZAR LEZCANO, M.S.(2000) "Cálculo de parámetros distribucionales y la función de Kullback-Liebler en redes de acceso público a la información de la República Argentina." Reunión de Comunicaciones Científicas y Técnicas de la Secretaría de Ciencia y Técnica de la UNNE. Rcia. http:// www1.unne.edu.ar/cyt/2000/7_tecnologicas/t_pdf/t_046.pdf

- GOUSSAL, D.; UDRíZAR LEZCANO, M.S. (2001) "Análisis paramétrico de área piloto de distribuciones de puntos de presencia en redes de acceso público a la información." Reunión de Comunicaciones Científicas y Técnicas de la Secretaría de Ciencia y Técnica de la UNNE. Rcia. http:// www1.unne.edu.ar/cyt/2001/7_tecnologicas/t_pdf/t_044.pdf

- ITU (1998). World telecommunication development report 1998 - universal access. Geneva.

- MORENO JIMÉNEZ, A. (2000), "Localización de la población y servicios de farmacia". En: Población y Espacio de la Comunidad de Madrid. Análisis y aplicaciones a nivel microgeográfico. Informe monográfico del Tomo 4 de la Estadística de Población de la Comunidad de Madrid. 2000.

- MORENO JIMÉNEZ, A., (2004) "Modelos de localización óptima de instalaciones y equipamientos". En: Bosque Sendra, J. y Moreno Jiménez, A. (Eds) Localización de equipamientos y SIG. Editorial RA-MA. Madrid, España.

\footnotetext{
${ }^{\text {i } E s t a ~ b a s e ~ s e ~ e n c u e n t r a ~ d i s p o n i b l e ~ e n ~ l a ~ p a ́ g i n a ~ d e ~ l a ~ E m p r e s a ~ A e r o t e r r a ~ q u e ~ d i s t r i b u y e ~ l o s ~ p r o d u c t o s ~ d e ~ E S R I . ~}$

${ }^{\mathrm{ii}}$ Esta base se encuentra disponible en la página de la Empresa Aeroterra que distribuye los productos de ESRI.

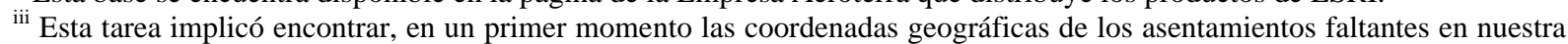
base original, elaborar un archivo compatible con un Sistema de Información Geográfica para que reconozca a las coordenadas como un archivo de puntos, convertir las coordenadas geográficas (definidas en un Sistema de Referencia) a coordenadas planas (definidas en un Sistema de Representación Cartográfica) y luego integrarlas a los más de quinientos departamentos para poder realizar los análisis requeridos.

iv MORENO JIMENEZ (1999) lo expresa como la relación entre los cocientes determinados por la proporción de oferta/recursos en cada punto de oferta con relación al total de oferta de la región estudiada y la proporción de demanda, es decir la proporción de demanda/usuarios, es decir la población de cada punto de demanda en relación al total de población/usuarios en todos los puntos de demanda del área estudiada.
} 